optimistic. Questionnaires can be and are produced that, for practical purposes, produce equivalent responses. Further research will resolve lingering reservations. To some extent the positive and practical tone of the work described is determined not just by its commercial origins but also by the fact that most work has been conducted in relatively compliant and homogeneous sections of European and North American societies. There are a few intellectual doubts reported in the volume. Naughton and Wiklund report evidence that the widely used Centers for Epidemiologic Studies - Depression (CES-D) scale does not produce patterns of depressive symptoms in some Asian American groups consistent with other cultural groups. Guyatt briefly suggests that the field reflects the "cultural hegemony" of middleclass American concerns before going on to suggest pragmatic research solutions. On the whole, similarities between cultures are far more apparent in this book than differences. It offers a powerful challenge to moral relativists, although the message is implicit, this not being the intention of the contributors.

While such work is confined to examining non-specific effects of drugs in the context of international randomised controlled trials, the cross-cultural thrust of much HRQOL may remain relatively uncontroversial. The current volume clearly, and in a most scholarly way, demonstrates the scientific caution and care of this new approach. If like the QALY, international assessments of HRQOL become involved in issues of choice about human life and resource allocation, we can expect a wider and more heated debate.

RAY FITZPATRICK Nuffield College, Oxford

\section{Good Clinical \\ Practice and Ethics in European Drug Research}

Edited by Peter Bennett, Bath, Bath University Press, 1994, 159 pages, $£ 30$.

This edited collection arose out of a symposium, held in 1993, that looked at the impact of European
Community guidelines on good clinical practice (EC GCP) in European drug research. The EC GCP sets out a uniform standard for the ethical conduct of clinical trials. The collection examines the effect of applying this uniform standard and details the complexities that exist in the review process due to the large number of ethics committees that have been formed in the member states.

The proliferation of ethics committees is a pressing problem for multicentred trials and some fascinating case studies are presented that graphically highlight both the time-consuming complexity of multi-centred applications and the often inadequate scientific and ethical evaluation of the intended trial. One team stated that even after their application was considered by 68 ethics committees in 12 European countries "certain basic questions and points concerning the ethics of the trial were not covered by any of the ethics committees" (page 64 ). The ethics committees also omitted to consider many of the points that are explicitly referred to in the EC GCP guidelines. Notably, information that an investigator was in a position to undertake the study was neither sent nor requested by any of the ethics committees and many of the committees not familiar with the drugs involved in the trial did not request further information, even though the existence of this information was referred to in the protocol. The team concluded: "It is difficult to understand on what basis these ethics committees were able to judge the relative benefits of ... [the] therapy" (page 58). This raises the issue of the effectiveness of any European GCP guidelines if there is no structure in place to ensure that committees rigorously apply these guidelines.

Some of the problems created by multi-centred trials could be addressed by a cross-Europe ethics committee that works in tandem with the local ethics committees. To provide this function the European Ethical Review Committee was set up in the late 1970 s and draws its membership from different countries in Europe to give a supranational ethics review. The work and role of this committee is considered in the book.

The contributions are largely from medics and one concern of the book is to give an account of the ethical review process in a number of countries (Denmark, the Nordic countries, Eastern Europe and the USA). This general overview provides a valuable insight into international differences. The collection also includes essays addressing: general issues in the ethical consideration of informed consent in clinical drug trials, ethical issues of adverse effects, and the problems created by special groups such as psychiatric and cancer patients. Furthermore, there is a discussion of how important it is that the membership of ethics committees fully understand the scientific issues raised by research protocols. This discussion concludes with a useful consideration of what kind of education should be provided for the membership to enhance their understanding of both the scientific and ethical aspects of research protocols.

As an appendix to the book the GCP for clinical trials on medicinal products in the European community is included in full (a useful reference for practitioners). The guidelines begin by stating that "the Declaration of Helsinki is the accepted basis for clinical trial ethics" (page 141). This gives an indication of the overall spirit of the guidelines but, as pointed out by one of the contributors, Oliver Guillod, this can give rise to a certain amount of ambiguity. There are points where the two guidelines conflict, for instance the Helsinki Declaration allows for conduct of clinical research without informed consent (principle 1.II) whereas the EC GCP does not. It is submitted that only one set of guidelines should be kept and this should be the EC GCP as, in Guillod's opinion, it is far better than the Helsinki Declaration.

This collection is a useful guide to how the ethical review process works in practice and provides an illuminating insight into regional differences. The consideration of the ethical dimensions of clinical research in a working context is an invaluable exercise, as often the abstract reiteration of ethical principles does not reflect the complexity of practical decision-making.

LUCY FRITH Department of Primary Care, University of Liverpool

\section{Chronic Illness: From Experience to Policy}

Edited by S K Toombs, D Barnard and R A Carson, Bloomington and Indianapolis, Indiana University Press, 1995, 221 pages, £23.50. 